\title{
O Desenvolvimento da Noção de Tempo como Integração da Distância e da Velocidade
}

\author{
Antonio Roazzi $i^{1}$ \\ Universidade Federal de Pernambuco \\ José Aires de Castro Filho \\ Universidade Federal do Ceará
}

\begin{abstract}
Resumo
$\mathrm{O}$ artigo investiga como surge e se desenvolve o conceito de tempo como integração da distância e da velocidade em uma amostra de 46 crianças de sete a dez anos de idade que estudavam em uma escola da rede pública de ensino. Os resultados diferem de achados anteriores porque mostram que mesmo as crianças de dez anos não utilizaram uma regra de divisão para realizar os julgamentos sobre tempo. Estes resultados apontam mais para diferenças em termos de experiência sociocultural e escolar do que para diferenças transculturais no desenvolvimento da noção de tempo como integração da distância e da velocidade.

Palavras-chave: Conceito de tempo; Piaget; cultura; escolarização.
\end{abstract}

The Development of the Concept of Time as Integration of Distance and Speed

\begin{abstract}
This study aimed at verifying how the concept of time as an integration of speed and distance emerged and developed in a sample of 46 seven to ten year-old public school children. Results were different from previous studies because even ten yearsold children did not use a division rule to achieve judgements about time. These results are interpreted as due more to differences in social, cultural, and school experience than to cross-cultural differences in the development of the notion of time as integration of distance and speed.

Keywords: Concept of time; Piaget; culture; schooling.
\end{abstract}

Os conceitos de tempo, distância e velocidade são utilizados por todas as pessoas em qualquer atividade cotidiana. Ao atravessarmos a rua, ao corrermos para pegar uma bola, ao calcularmos o tempo que levaremos para chegar em algum lugar e em muitas outras ocasiões, estamos constantemente integrando e avaliando, de forma intuitiva, as informações sobre tempo, distância e velocidade. Esses conceitos vêm sendo formalmente estudados há muito tempo pela Física. A preocupação em descrever os movimentos e compreendê-los através de leis levou à formulação de várias teorias. A mecânica newtoniana propunha o tempo como uma noção fundamental e definia a velocidade como uma função da distância percorrida num dado intervalo de tempo (Velocidade $=$ Distância $/$ Tempo). Já a teoria da relatividade, proposta por Einstein, sugere que tempo e

${ }^{1}$ Endereço para correspondência: Universidade Federal de Pernambuco, $\mathrm{CFCH}, 8^{\circ}$ andar, Cidade Universitária, Rua Acadêmico Hélio Ramos, s/n, 50670-901, Recife, PE. Fone: (81) 32718272. Fax: (081) 32711843. E-mail: roazzi@npd.ufpe.br

${ }^{2}$ Endereço para correspondência: Universidade Federal do Ceará, Rua Dom Expedito Lopes, 2360/1302, 60-135-410, Fortaleza, CE. Fone: (85) 461.1474. Fax: (85) 2833926.E-mail: j.castro@ufc.br velocidade são definidos em função do outro (e com relação a pontos fixos), pelo menos para velocidades próximas à da luz (Frota, 1995).

Mas o que acontece em nível psicológico? Como os conceitos de tempo, distância e velocidade surgem e se desenvolvem? Será que se desenvolvem de maneira separada ou conjunta? Esses conceitos inicialmente estariam mais ligados à Física newtoniana ou à teoria da relatividade? Questões desse tipo foram propostas por Albert Einstein a Jean Piaget, em 1928. Algumas respostas foram encontradas por Piaget (1946a, 1946b) e outras novas surgiram, das quais várias ainda permanecem sem explicação (Frota, 1995).

Nesse trabalho, buscaremos inicialmente uma reconstituição das pesquisas nessa área, iniciando por Piaget e mostrando os desdobramentos e questionamentos feitos por pesquisadores como Levin, Siegler e Wilkening, entre outros. Em seguida, descreveremos um experimento que buscou contribuir para a compreensão de como esses conceitos se desenvolvem em crianças.

Em seus trabalhos sobre a noção de tempo, Piaget realizou estudos sobre a sucessão dos acontecimentos percebidos e sobre a idéia de simultaneidade (Piaget, 
1946a, 1946b). O método empregado nos dois estudos foi muito semelhante e consistiu em dois carrinhos que se deslocavam de um ponto a outro no espaço. Os carrinhos podiam partir do mesmo ponto ou de pontos diferentes, ter a mesma velocidade ou velocidades diferentes e ainda andar durante o mesmo tempo, ou tempos diferentes. Piaget perguntava à criança, então, qual carrinho tinha andado mais tempo, ou qual tinha maior velocidade, ou ainda qual tinha percorrido a maior distância.

Piaget descobriu que as crianças que se encontravam no período pré-operacional não conseguiam coordenar as sucessões temporais e espaciais e também não julgavam que os movimentos eram simultâneos. Em geral, confundiam os conceitos de tempo, distância e velocidade, não distinguindo muito bem um conceito do outro. Ao fazerem julgamentos sobre tempo, por exemplo, afirmavam que a duração era sempre proporcional ao caminho percorrido. Piaget (1946a) concluiu que o conceito de tempo somente é adquirido quando a criança já tem a noção de velocidade sob uma forma operatória, isto é, como uma relação entre o espaço percorrido e essa dimensão (tempo), comum às diferentes velocidades. Piaget (1946a) considerou ainda que os conceitos de tempo, distância e velocidade são construtos, i.e., não estão presentes a priori na mente da criança, mas requerem uma construção ontogenética. Inicialmente (estágio I), a criança pré-operacional julga somente levando em consideração o ponto de parada. No estágio II, a criança começa a considerar outros fatores, como o ponto de partida. É o período em que inicia a descentração, mas ainda intuitivamente. Finalmente, no estágio III, a criança obtém o pleno domínio desses conceitos.

Os achados de Piaget têm sido questionados e criticados sob diversos aspectos: (1) experimental: questionando o procedimento experimental (Levin, 1979); (2) cultural: questionando a influência de aspectos socioculturais (Bovet, 1975; Mori, 1976); (3) teórico: questionando os pressupostos de Piaget sobre os experimentos (Siegler \& Richards, 1979); (4) metodológico: questionando o próprio experimento (Wilkening, 1981). A seguir, iremos comentar alguns desses estudos.

Levin (1979) afirmou que o problema encontrado na tarefa de tempo estaria no número de variáveis de interferência presentes no experimento. A fim de comprovar essa hipótese, Levin comparou a performance de 144 crianças em três tarefas nas quais era pedido para que elas julgassem, entre dois "eventos", aquele que demorava mais. Os eventos foram chamados: tempo de pausa (o tempo de duas bonecas dormindo), tempo de rotação (o tempo de dois pares de figuras girando sobre um eixo) e tempo linear (o tempo de dois carros de brinquedo se deslocando sobre uma pista). Com base nos resultados, Levin (1979) concluiu que havia uma influência dessas variáveis, embora as diferenças entre o tempo de pausa e o rotacional não fossem tão acentuadas quanto às diferenças entre o tempo de pausa e o linear e entre o tempo rotacional e o linear.

Com respeito ao aspecto cultural, Mori (1976) questionou a universalidade dos achados de Piaget. O autor confrontou o desempenho de crianças japonesas com crianças tailandesas, num experimento de comparação de velocidade e duração temporal. Mori (1979) concluiu que as crianças tailandesas têm mais facilidade de fazer julgamentos temporais, porque possuem no vocabulário palavras distintas para significar "veloz" (fast) e "adiantado" (early). Diferentemente, as crianças japonesas só possuem na sua língua uma palavra (bayai) para ambos os significados. Mori defendeu que a construção desses conceitos depende consideravelmente das diferenças existentes entre os idiomas.

Bovet (1975) realizou um estudo no qual replicava algumas das situações experimentais utilizadas por Piaget (1946a) em um meio africano, com adultos não-escolarizados e adolescentes escolarizados e não-escolarizados, de ambos os sexos. Bovet (1975) encontrou que somente os adultos homens e os adolescentes não-escolarizados eram capazes de realizar inferências sobre noções temporais. À luz da teoria piagetiana, tais achados indicam um atraso na chegada ao estágio das operações concretas, uma vez que os sujeitos utilizados por Piaget apresentavam essas noções por volta dos dez anos de idade.

Outro questionamento aos estudos de Piaget foi feito por Siegler e Richards (1979). Segundo os autores, embora Piaget afirmasse que a criança domina os três conceitos (tempo, distância e velocidade) de forma simultânea, ele nunca examinou a mesma criança nas três tarefas. Siegler e Richards (1979) propuseram, então, uma tarefa que foi aplicada, de forma equivalente, aos mesmos sujeitos e que consistiu num teste de 24 itens, nos quais eram manipuladas as seis dimensões físicas envolvidas (velocidade, distância, ponto final, ponto inicial, tempo final e tempo inicial). Todos os itens basearam-se no movimento de dois trens elétricos de brinquedo. Os participantes foram 36 crianças de uma escola de classe média de Pittsburg, EUA (doze do pré-escolar, doze da terceira série e doze da sexta) e doze universitários. A cada item, o sujeito era solicitado a responder qual dos trens (azul ou vermelho) andava mais (ou por mais tempo, ou com maior velocidade) ou se andavam a mesma distância (ou o mesmo tempo, ou com a mesma velocidade). Uma das principais conclusões do estudo foi que parece haver uma forte correlação entre as idades 
dos sujeitos e as regras utilizadas para resolver os problemas. Além disso, os autores apontaram para o fato de que o pleno domínio desses conceitos parece surgir mais tarde do que Piaget havia predito.

Wilkening (1981) criticou os métodos anteriormente empregados pelos pesquisadores para investigar as noções de tempo, espaço e velocidade, quando a criança tem dois objetos e tem que concluir qual dos dois andou por mais tempo, andou a maior distância, ou andou com a maior velocidade. Segundo o autor, a principal objeção a ser feita sobre esse método, denominado método de escolha, é que ele não permite observar se o sujeito está usando alguma regra algébrica, i.e., aplicando uma operação matemática (soma, multiplicação, divisão) com as quantidades envolvidas (distância, tempo e velocidade). Uma criança pode concluir qual objeto andou durante mais tempo sem considerar a relação entre distância, velocidade e tempo.

Wilkening (1981) desenvolveu um método, chamado método de mensuração funcional, com o objetivo de lidar com regras para integrar informações de diferentes dimensões. $\mathrm{O}$ autor utilizou sujeitos com três grupos de idades: cinco anos, dez anos e adultos entre dezessete e trinta e cinco anos ( $m=25$ anos). Os sujeitos foram submetidos a três tarefas, nas quais estavam envolvidas as variáveis tempo, distância e velocidade. Em cada tarefa, era solicitado ao sujeito que desse uma resposta sobre uma variável, tendo informações sobre as outras duas.

$\mathrm{Na}$ tarefa em que foi testada a integração da velocidade e da distância para responder sobre o tempo, Wilkening (1981) encontrou que os indivíduos foram capazes de integrar a velocidade e a distância, através de uma regra algébrica, para fazerem julgamentos sobre o tempo, embora somente os sujeitos com dez anos e os adultos tenham utilizado corretamente a regra de divisão (tempo $=$ distância/velocidade), enquanto os sujeitos de cinco anos utilizaram uma regra de subtração (tempo= distância-velocidade).

Acredolo, Adams e Schmid (1984) realizaram um estudo no qual combinaram o método de integração funcional, proposto por Wilkening (1981), com o método de escolha utilizado nos experimentos anteriores (Piaget, 1946a, 1946b, Siegler \& Richards, 1979). O experimento foi bastante semelhante ao de Wilkening. Contudo, as crianças não foram solicitadas a analisar quanto tempo, ou com qual velocidade, ou com qual distância, animais haviam corrido, o que implica em um raciocínio quantitativo. Em cada tarefa, ao invés de um animal, eram apresentados dois animais e a criança era solicitada a afirmar qual das proposições era correta, se os animais andaram durante um mesmo tempo (ou com a mesma

Psicologia: Reflexão e Crítica, 2001, 14(3), pp. 497-503 velocidade; ou a mesma distância) ou se um animal andou durante mais tempo (ou mais rápido; ou uma distância maior) do que o outro. Segundo os autores, essa forma de perguntar implica em um raciocínio qualitativo, mas do ponto de vista da lógica o objetivo era determinar se as crianças seriam capazes de perceber, na integração de diferentes dimensões, relações diretas e inversas. Acredolo, Adams e Schmid (1984) concluíram que as relações inversas entre velocidade e duração são atingidas mais tarde que as relações diretas entre velocidade e distância e entre duração e distância. Segundo os autores: "Embora a maioria das crianças de quarta e quinta série parecia entender as relações entre todos os possíveis pares das três dimensões, elas não pareciam integrar essas relações espontaneamente" (Acredolo, Adams \& Schmid, 1984, p. 2151).

Mais recentemente, Roazzi, Castro-Filho e Santos (1994) realizaram um estudo com 45 sujeitos brasileiros (cinco anos, dez anos e adultos) de nível socioeconômico médio, a partir do experimento de Wilkening (1981). Os autores investigaram uma possível explicação, dada pelo próprio Wilkening, de que as crianças de cinco anos julgariam incorretamente o tempo por estarem, aparentemente, muito distraídas com o botão e com o estímulo da distância. Os autores acrescentaram, então, no planejamento experimental, uma condição verbal. Os resultados indicaram não haver diferença entre a condição manual e a verbal. Ao mesmo tempo, indicaram que as regras de integração dependem da idade, confirmando os dados principais encontrados por Wilkening (1981) com sujeitos alemães. De fato, enquanto os dois grupos de sujeitos mais velhos forneciam respostas de acordo com regras de divisão (tempo= distância/velocidade), as crianças de cinco anos de idade utilizavam regras muito mais simples. Assim, esses resultados indicam que a aquisição do conceito de tempo como integração da distância e da velocidade ocorre entre as idades de cinco e dez anos.

$\mathrm{Na}$ presente investigação objetivamos estabelecer com mais precisão o período de aquisição desse conceito de tempo, como integração da distância e da velocidade, visando averiguar como ele surge e se desenvolve. Em que idade começa a haver integração das informações? Antes dessa idade, as crianças realizam o julgamento baseado em quais aspectos? Enfim, o meio sociocultural exerce alguma influência sobre esse julgamento? No estudo descrito a seguir, propomo-nos elucidar essas questões. Procuramos, ainda, modificar o contexto da tarefa, de modo a torná-la um pouco mais compreensível para as crianças. Além disso, crianças de escola pública foram avaliadas para serem realizadas comparações sobre a influência da experiência sociocultural sobre os julgamentos de tempo. 


\section{Participantes}

A amostra foi composta por 46 crianças, sendo doze de sete anos $(m=7,4 ; d p=2,71)$, doze de oito anos $(m=8,3$; $d p=2,76)$, onze de nove anos $(m=9,4 ; d p=4,08)$ e onze de dez anos $(m=10,4 ; d p=2,69)$. As crianças estudavam em uma escola da rede de ensino público da cidade de Fortaleza.

\section{Materiais}

Foram utilizados os seguintes materiais: um painel de três metros de comprimento por um metro de largura, no qual estavam desenhadas uma casinha para cada animal (tartaruga e gato) e três jaulas (distâncias de 50 cm, 100 $\mathrm{cm}$ e $150 \mathrm{~cm}$ ) onde seria colocada a comida de acordo com a refeição (pela ordem, café da manhã, almoço e jantar); dois pedaços de cartão de 10x16 cm, com desenho de pratos com alimentos para os animais (leite para o gato e folha para a tartaruga); um gravador com uma fita cassete, na qual estava gravado o barulho de uma sirene; um interruptor, que permitia ligar e desligar o gravador; um cronômetro.

\section{Procedimentos}

Os sujeitos foram testados, individualmente, na presença de dois experimentadores. Inicialmente, o experimentador explicava à criança que aquele painel era um jardim zoológico e lhe perguntava quais eram aqueles bichos e qual o mais rápido e o mais lento. Em seguida, o experimentador afirmava que a criança deveria ajudá- lo a alimentar os animais e dizia que aqueles animais comiam somente três vezes por dia: no café da manhã, no almoço e no jantar. $\mathrm{Na}$ hora da refeição, o empregado do zoológico colocava o prato de comida dentro de uma grade para nenhum outro bicho comer e apertava um botão que tocava uma sirene e abria a porta da casinha do bicho. O bichinho estava com tanta fome que corria o mais rápido que podia para pegar sua comida. $\mathrm{O}$ experimentador então afirmava: "Quando o animal chegar na frente da grade você vai ajudá-lo, apertando o botão que abre a porta da grade. Mas a porta só abre uma vez e fecha bem rápido. Se você abrir antes do bichinho chegar, a porta se fecha e ele vai perder a comida, vai continuar com fome e vai ficar bem triste. Então presta bem atenção para só apertar o botão quando o bichinho chegar na porta". O experimentador em seguida colocava o prato de comida e ligava o gravador. Cada prato de comida era colocado duas vezes em cada posição $(50 \mathrm{~cm}, 100 \mathrm{~cm}$ e $150 \mathrm{~cm})$, totalizando doze estimativas de tempo para cada sujeito. A ordem de apresentação dessas estimativas era também controlada. A leitura do tempo era feita por um outro experimentador.

\section{Resultados}

$\mathrm{Na}$ análise tomamos como base a média das estimativas de tempo dos sujeitos em função da distância e da velocidade. Um padrão esperado seria que os sujeitos integrassem a distância e a velocidade, usando uma

Tabela 1. Média $(m)$ e Desvio-Padrão $(d p)$ dos Julgamentos do Tempo como Função da Velocidade e Distância por Cada Faixa Etária

\begin{tabular}{|c|c|c|c|c|c|c|}
\hline \multirow{3}{*}{ Idade (anos) } & \multirow{3}{*}{ Distância } & \multicolumn{5}{|c|}{ Velocidade } \\
\hline & & \multicolumn{2}{|c|}{ Tartaruga } & \multicolumn{2}{|c|}{ Gato } & \multirow{2}{*}{$\frac{\text { Total }}{m}$} \\
\hline & & $m$ & $d p$ & $m$ & $d p$ & \\
\hline \multirow[t]{4}{*}{7 anos } & 50 & 12,63 & 13,40 & 9,62 & 8,08 & 11,13 \\
\hline & 100 & 11,66 & 10,31 & 10,76 & 8,24 & 11,21 \\
\hline & 150 & 11,45 & 7,18 & 12,45 & 8,06 & 11,95 \\
\hline & Total & 11,91 & & 10,94 & & 11,43 \\
\hline \multirow[t]{4}{*}{8 anos } & 50 & 11,99 & 4,90 & 12,25 & 5,08 & 12,12 \\
\hline & 100 & 16,95 & 7,09 & 14,83 & 6,55 & 15,89 \\
\hline & 150 & 18,63 & 8,86 & 18,76 & 8,61 & 18,70 \\
\hline & Total & 15,85 & & 15,28 & & 15,57 \\
\hline \multirow[t]{4}{*}{9 anos } & 50 & 8,74 & 5,22 & 8,00 & 4,48 & 8,37 \\
\hline & 100 & 10,20 & 5,37 & 9,65 & 4,61 & 9,93 \\
\hline & 150 & 11,75 & 5,85 & 11,22 & 5,42 & 11,49 \\
\hline & Total & 10,23 & & 9,62 & & 9,93 \\
\hline \multirow[t]{4}{*}{10 anos } & 50 & 11,40 & 10,62 & 8,41 & 7,13 & 9,91 \\
\hline & 100 & 13,93 & 10,28 & 10,27 & 7,51 & 12,10 \\
\hline & 150 & 15,89 & 8,66 & 13,81 & 11,13 & 14,85 \\
\hline & Total & 13,74 & & 10,83 & & 12,28 \\
\hline
\end{tabular}


estimativa baseada numa regra de divisão $(T=D / V)$, levando ambas as quantidades em consideração. Uma outra possibilidade seria os sujeitos levarem em consideração apenas uma das quantidades (distância ou velocidade) ou as duas quantidades, mas sem integrá-las.

A Tabela 1 mostra a média das estimativas de tempo dos três grupos de sujeitos em função da distância e da velocidade. Observamos que, em cada faixa etária, a distância parece ser mais bem compreendida do que a velocidade. Uma maior distância sempre implica um tempo médio mais longo - relação diretamente proporcional, à exceção das crianças de sete anos de idade, com a tartaruga.

Observamos uma relação inversamente proporcional para a variável velocidade (uma maior velocidade implicando um tempo inferior). A tartaruga apresenta sempre um maior tempo do que o gato, para todas as idades. É importante notar que esta diferença encontrase mais acentuada para as crianças de dez anos de idade, enquanto para as crianças de sete, oito e nove anos de idade, ela é muito pequena. Portanto, podemos concluir que somente aos dez anos de idade a integração da distância e da velocidade é realizada.

Para melhor visualizar a existência de efeitos interativos da relação entre as variáveis velocidade/distância, as médias destas variáveis foram representadas na Figura 1. Verificamos que, para os quatro grupos de idade, não se observa efeitos interativos do tipo abertura de leque das
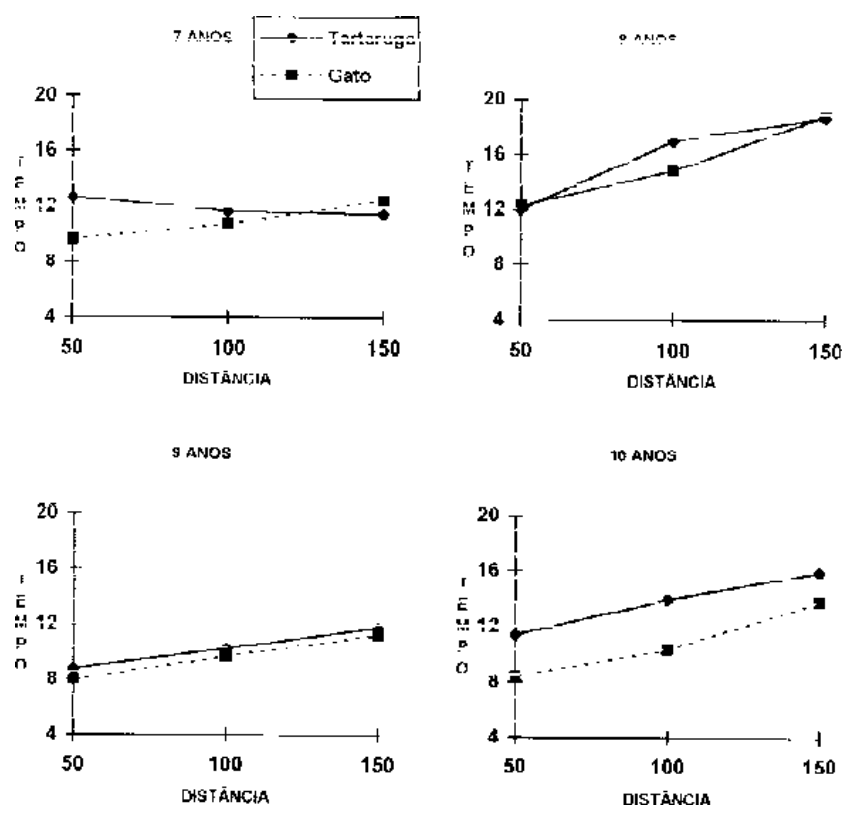

Figura 1. Gráficos dos julgamentos do tempo como função da velocidade e distância por cada faixa etária linhas, o que implicaria que nos gráficos quanto maior a velocidade, menor a inclinação da reta. Isso se explica porque o tempo pode ser calculado pela equação Tempo $=$ Distância/Velocidade $(T=D / V)$. Como para cada animal a velocidade é constante, o cálculo do tempo pode ser descrito como uma função linear da distância para cada animal, $T=K^{*} D, K=1 / V$. Portanto, quanto maior a velocidade do animal, menor o valor da constante $\mathrm{K}$ e menor a inclinação da reta. Por outro lado, podemos observar um pequeno efeito interativo inverso nas crianças de sete anos de idade, o que corrobora a idéia de que, nessa idade, as crianças ainda não realizam uma integração.

Para verificar os níveis de significância dessas diferenças, os dados foram analisados através da Análise de Variância, para cada faixa etária, em um planejamento experimental misto com medidas repetidas nas condições: distância (3: $50 \mathrm{~cm}, 100 \mathrm{~cm}$ e $150 \mathrm{~cm})$ e velocidade $(2$ : Tartaruga e Gato). O estudo não mostrou diferença significativa em relação ao sexo dos sujeitos $[F(1,43)=1,17 ; p=$ n.s. $]$ e à ordem de realização das tarefas $[F(1,43)=74,11 p=$ n.s. $]$.

Confirmando as conclusões acima apresentadas, não observamos nenhum efeito interativo Velocidade $\mathrm{x}$ Distância. A falta de um tipo de interação representando uma configuração de abertura em leque entre as variáveis indica como cada faixa etária não integrou a distância e a velocidade, utilizando um modelo de divisão (Tempo=Distância/Velocidade). $\mathrm{O}$ alto valor do desvio padrão, para algumas situações, é uma evidência adicional de que as crianças não usaram um modelo de divisão. Esperávamos um padrão nas respostas das crianças, o que tornaria o desvio padrão menor.

Foram encontrados efeitos principais somente na variável Velocidade, nas crianças de dez anos de idade $[F(1,11)=6,85 ; p<0,031]$. Portanto, nessa faixa etária as crianças começam a levar a velocidade em consideração. Em relação à variável Distância, um efeito principal foi observado em crianças de oito, nove e dez anos de idade $[F(2,22)=12,04 ; p<0,001 ; F(1,11)=5,66, p<0,012$ e $F(2,22)=49,71, p<0,001$, respectivamente], mas não nas crianças de sete anos de idade, o que corrobora ainda mais a noção de que as crianças de sete anos não levam a distância em consideração. Apenas a partir de oito anos, a distância começa a ser considerada.

\section{Discussão}

Os resultados deste estudo permitem levantar uma série de discussões. Inicialmente, observamos que esses resultados divergem parcialmente dos estudos anteriores de Wilkening (1981) e Roazzi, Castro-Filho e Santos 
(1994), ao mostrar que mesmo as crianças de dez anos não utilizaram uma regra de divisão para realizar os julgamentos sobre tempo. Podemos explicar esse fato pelas diferenças entre as duas amostras. Enquanto as crianças dos dois primeiros estudos eram oriundas de famílias de nível socioeconômico médio (escola particular), as crianças deste estudo pertenciam a famílias de nível socioeconômico baixo (escola pública). Dado que no Brasil existem diferenças em termos de escolarização (tanto quantitativas como qualitativas), em função do tipo de escola freqüentada, tal resultado não representa surpresa uma vez que estudos anteriores sobre o próprio conceito de tempo já mostraram a influência da variável escolarização, indicando uma defasagem na chegada de sujeitos ao raciocínio operatório concreto (Bovet, 1975). Esses resultados apontam mais para diferenças em termos de experiência sociocultural e escolar do que para diferenças transculturais (no caso de crianças alemãs versus crianças brasileiras) no desenvolvimento da noção de tempo como integração da distância e velocidade. Este desenvolvimento não varia em função de diferenças culturais, pelo menos quando comparamos crianças brasileiras e alemãs. A escolarização parece exercer maior influência sobre o desenvolvimento desses conceitos.

Um outro dado interessante é a seqüência como esses conceitos se formam. Observamos que as crianças inicialmente apresentam diferenças no julgamento, considerando a distância percorrida, mas não a velocidade. Em seguida, a crianças utilizam a distância e a velocidade, mas sem integrá-las através da regra de divisão. Achados semelhantes foram apontados por Wilkening (1981) e Roazzi e colaboradores (1991).

Resultados similares foram comentados por outros autores (Acredolo, Adams \& Schmid, 1984; Siegler \& Richards, 1979). Acredolo e colaboradores observaram que as três dimensões (tempo, distância e velocidade) diferem com relação à atenção que uma dimensão desperta em relação às outras. Segundo esses autores, a dimensão distância é a mais saliente e, portanto, a que mais interfere quando as crianças fazem julgamentos sobre velocidades e durações. Também Siegler e Richards (1979) apontam para a pouca diferenciação entre o conceito de tempo e distância. Em seu estudo, as crianças mais novas utilizaram uma regra baseada somente na distância para realizar os julgamentos. Devemos considerar que Acredolo e colaboradores (1984) e Siegler e Richards (1979) enfocaram aspectos diferentes do conceito de tempo, mais ligado à compreensão lógica do conceito, enquanto em nossos estudos e no de Wilkening 1981, enfocamos a compreensão algébrica ou de quantificação. Apesar disso, achamos que a generalização é válida, embora ainda precise ser confirmada em estudos posteriores.
Finalmente, gostaríamos de levantar uma questão ainda não esclarecida nesses estudos. Wilkening (1981) sugere que a integração ocorre através de uma regra algébrica de subtração, para as crianças mais novas, e de divisão para as crianças mais velhas e os adultos. Enquanto a regra de divisão aparece muito claramente nos gráficos (uma reta com inclinação quase constante), o mesmo não acontece com a regra de subtração. Se observarmos os gráficos de Wilkening (1981) e os gráficos mostrados neste estudo (Figura 1), não seremos capazes de deduzir o uso dessa regra. Duas outras evidências apontam para essa conclusão. A primeira é que, nas crianças mais novas, somente foram encontradas diferenças de julgamento em relação à distância. Portanto, essas crianças não poderiam utilizar uma regra algébrica (de subtração ou divisão), uma vez que só levam em consideração uma dimensão. Poderíamos esperar no máximo uma regra qualitativa quanto maior a distância, maior o tempo gasto - dado já apontado nos estudos de Acredolo e colaboradores (1984) e Siegler e Richards (1979).

Uma outra evidência para essa conclusão é a de que as velocidades nos estudos de Wilkening (1981), Acredolo e colaboradores (1984), Roazzi e colaboradores (1991) são dadas de forma apenas qualitativa, ou seja, as crianças apenas são informadas de que existem animais com diferentes velocidades (e todas concordam com esse dado), mas não é dada nenhuma pista de quanto é essa velocidade. Enquanto a distância está evidente para a criança medir e o tempo é medido de forma indireta (pelo tempo de algum sinal sonoro), em nenhum momento a criança vê os animais se deslocando com sua velocidade. Isto pode tornar a tarefa mais difícil para as crianças mais novas, embora as mais velhas consigam realizá-la, talvez estabelecendo uma velocidade para cada animal e guiando-se por esse padrão.

Thompson (1994) investigou uma estudante de dez anos da quinta série em sessões de ensino que tinham como base um micromundo computacional. Nesse programa, as dimensões velocidade, tempo e distância eram sempre dadas de forma numérica e o sujeito era solicitado a tirar conclusões desses dados. Thompson também encontrou uma certa confusão inicial entre os conceitos de tempo, distância e velocidade e afirmou que o método utilizado pelo sujeito para determinar a quantidade de tempo necessária para percorrer uma dada distância a uma certa velocidade surge de uma noção primária de velocidade, como um comprimento de velocidade em uma unidade de tempo (e.g., uma unidade de 40 pés em um segundo). Segundo o autor, somente no final do experimento é que o sujeito passou a apresentar a compreensão da velocidade como uma razão entre 
distância e tempo. Sugerimos, portanto, que futuras pesquisas investiguem como as crianças realizariam os julgamentos, caso a dimensão velocidade fosse apresentada de maneira quantitativa, ou seja, as crianças veriam os objetos se deslocando e a velocidade dos objetos fosse bem diferente, algo como um homem a pé, um homem de carro e um homem num avião a jato.

\section{Referências}

Acredolo, C, Adams, A. \& Schmid, J. (1984). On the understanding of the relationships between speed, duration, and distance. Child Development, 55, 2151-2159.

Bovet, M. (1975). Étude piagétienne de quelques notions spatio-temporelles dans un milieu african. International Journal of Psychology, 10, 1-17.

Frota, P. R. O. (1995). Integração da distância como função da velocidade e do tempo em crianças de duas diferentes escolas. Dissertação de Mestrado não publicada. Universidade Federal do Piauí. Teresina, Piauí.

Levin, I. (1979). Interference of time-related and unrelated cues with duration comparisons of young children: Analysis of Piaget's formulation of the relation of time and speed. Child Development, 50, $469-477$
Mori, I. (1976). A cross-cultural study on children's conception of speed and duration: A comparison between Japanese and Thai children. Japanese Psychological Research, 18(3), 105-112.

Piaget, J. (1946a). Le developpement de la notion du temps chez le enfant. Paris: Presses Universitaires de France.

Piaget, J. (1946b). Le notions de mouvement et de vitesse chez l'enfant. Paris: Presses Universitaires de France.

Roazzi, A., Castro-Filho, J. A. \& Santos, A. R. R. (1994). O tempo como integração da distância e da velocidade. Psicologia: Teoria e Pesquisa, 10, 4958.

Siegler, R. S. \& Richards, D. D. (1979). Development of time, speed and distance concepts. Developmental Psychology, 15(3), 288-298.

Thompson, P. W. (1994). The development of the concept of speed and its relationship to concepts of rate. Em G. Harel \& J. Confrey (Orgs.), The development of multiplicative reasoning in the learning of mathematics. Albany, NY: Suny Press.

Wilkening, F. (1981). Integrating velocity, time, and distance information: A developmental study. Cognitive Psychology, 13, 231-247.

Sobre os autores

Antônio Roazzi é PhD em Psicologia do Desenvolvimento pela University of Oxford, Inglaterra, Professor Adjunto do departamentode Psicologia da Universidade Federal de Pernambuco.

José Aires de Castro Filho é PhD em Educação Matemática pela University of Texas at Austin, EUA, Professor Adjunto do Departamento de Fundamentos da Educação da Universidade Federal do Ceará. 